\title{
Novel Framework for Navigation using Enhanced Fuzzy Approach with Sliding Mode Controller
}

\author{
T. Someswari ${ }^{1}$, Anil Kumar Tiwari ${ }^{2}$, Nagaraj R. ${ }^{3}$ \\ ${ }^{1,3}$ Department of Electrical \& Electronics Engg, The Oxford College of Engineering, India \\ ${ }^{2}$ Amity School of Engineering, Amity School of Engineering, India
}

\begin{tabular}{l} 
Article Info \\
\hline Article history: \\
Received Apr 2, 2018 \\
Revised Jul 18, 2018 \\
Accepted Aug 1, 2018 \\
\hline Keyword: \\
Fuzzy \\
Global positioning system \\
Inertial navigation system \\
Navigation \\
Sliding mode controller
\end{tabular}

Sliding mode controller

\begin{abstract}
The reliability of any embedded navigator in advanced vehicular system depends upon correct and precise information of navigational data captured and processed to offer trustworthy path. After reviewing the existing system, a significant trade-off is explored between the existing navigational system and present state of controller design on various case studies and applications. The existing design of controller system for navigation using error-prone GPS/INS data doesn't emphasize on sliding mode controller. Although, there has been good number of studies in sliding mode controller, it is less attempted to optimize the navigational performance of a vehicle. Therefore, this paper presents a novel optimized design of a sliding mode controller that can be effectively deployed on advanced navigational system. The study outcome was found to offer higher speed, optimal control signal, and lower error occurances to prove that proposed system offers reliable and optimized navigational services in contrast to existing system.
\end{abstract}

Copyright $(2018$ Institute of Advanced Engineering and Science. All rights reserved.

\section{Corresponding Author:}

T. Someswari,

Department of Electrical \& Electronics Engg,

The Oxford College of Engineering, Bangalore, India.

Email: someswarioxford@gmail.com

\section{INTRODUCTION}

With the increase of advancement in communication devices, there is an equivalent increase in an embedded navigation device too [1], [2]. In this regards, both the inertial navigation system (INS) and Global Positioning System (GPS) are considered as the prime actors for offering an effective navigational services [3], [4]. Also, found that more researches were exist for joint implementation of both INS as well as GPS. A closer look into such navigational system shows that navigational information offered by INS system is potentially associated with errors [5]. Although, such errors in navigational information of INS are highly short termed but it spontaneously degrades over a period of time. On the other hand, navigational information offered by GPS is considered to be highly stable along with better provision of precise data related to speed, location, and attitude [6]. It was also found that information related to all these physical and kinetic entities are provided by INS while information related to only location and speed is given by GPS.

There is also a potential dependency of both INS as GPS together as an effective and reliable navigational information cannot be offered by INS standalone and the main reason behind it is more occurances of errors associated with inertial sensors. It has also been seen that a continuous stream of navigational data can be offered by INS while data stream is always intermittent for GPS owing to various forms of signal interference causing outage [7], [8]. Therefore, 95\% of the existing research-based work has considered both INS and GPS for evolving up with a better form of navigational system. Usage of such joint navigational system can be seen in different forms of vehicle moving on land [9], water [10], and air [11]. There are also various forms of weapons (e.g. missiles) which uses such joint navigational system [12]. There is no denying the fact that various researches were available regarding this concern, but at the same time, it 
cannot be ignored that existing research approaches were not seem to successfully benchmark or claimed to offer superior navigational system [13]. Existing researchers are also attempting to construct cost effective INS-GPS navigational system where the prominent challenge is to precisely initialize the target angle owing to degraded working process of gyros or similar forms of other sensors. This is extremely essential problems to be addressed as there is an increasing trend of developing remotely driven vehicles. Hence, wrong information related to navigation system will render a collateral damage on such forms of smart vehicle. One of the effective mechanisms to address this problem could be by using sliding mode controller [14] which is the heart of every advance control system. For every complex and advanced vehicular system comes with a smart navigation that is capable of using both GPS and INS related signals.

There has been significant number of research work being carried out towards developing sliding mode control system; however, majority of them are associated with certain inherent problems of dealing with dynamic system. It was observed that existing approaches doesn't cater up the uncertainty demands of the dynamic system especially in transmission network. With majority of the work lacking benchmarking and non-inclusion of considering the case study of solving navigational problem, the sliding mode controller has become one of the less explored solutions toward enhancing navigation system using GPS and INS. Therefore, a novel technique is introduced to solve the navigational problems in GPS and INS. The review of existing researches and corresponding research problems is discussed in Section-2 and Section-3 respectively. Section-4 highlights about adopted methodology as solution along with illustration of algorithm design is given in Section-5. The performance analysis is discussed in Section-6 while Section-7 outlines the summary of the paper as conclusion.

\subsection{Background}

At present, there are various literatures focusing on designing an effective navigational system using INS as well as GPS, with unique methods and mechanism. Existing studies are more focused on incorporating better precision where the study led by Dacheng et al [15] proved enhanced sensitivity by minimizing Doppler frequency when navigational system of INS is integrated with GPS. Adoption strategy of filters is one unique approach in this research direction. According to Cho et al. [16], an enhanced Kalman filter would improve the navigational performance especially focusing on cost-effectiveness deployment viewpoint. Deployment of similar Kalman filter of extended type was seen in the work of Duong and Nguyen [17] for an effective estimation of physical navigational parameters e.g. attitude, speed, location, etc. Usage of Kalman filter was also seen in work discussed by Fang and Gong [18] where the prime objective was to incorporate a predictive scheme of an integrated navigational service.

The study was tested over real-time sensors mounted on aircraft to find that offers better accuracy. There was also research work which says that developing navigational system for underwater vehicle is really a challenging one. Such research is found in Lee et al [19] which is meant for minimizing the errors in navigational system especially with the GPS data. The work carried out by Li and Sun [20] has also used Kalman filter of extended form for improving the accuracy associated with navigational data. The works of $\mathrm{Wu}$ et al [21] have focused on developing a predictive algorithm using Kalman filter. The study also formulates a noise model for compensating the signals. Yan et al.[22] have also carried out work using Kalman filter .

Case study of flying object and its improvement of navigation data was researched by Nakanishi et al [23]. The authors have addressed the problem associated with reliability of data from GPS by implementing a centralized mechanism of sensor integration with an aid of updates obtained in asynchronous mode of communication. Case study of navigation of land vehicle has been considered in Qin et al. [24], where the Kalman filter as well as standard fuzzy inference system for minimizing errors in navigation system. Another author viz. Sun et al. [25] have carried out an experimental based analysis where a GPSINS based navigation system. It was also found that coupling among the navigational parameters significant affect the detection of signal. This issue has been researched by Jamal [26] by rectifying the errors associated with sensing during high speed of data capturing. According to author, tight coupling results in addressing these flaws. Another form of approach was the usage of machine learning schemes in navigational system. Usage of neural network was witnessed in the work of Jaradat and Hafez [27] where a regression-based approach was implemented for addressing the communication delay in navigation system. Existing system has also witnessed study towards identifying and addressing faults in navigational system. The work of Xin et al. [28] has used statistical-based approach for fault identification.

Although, there are various studies towards improving navigational performance, but an efficient controller can be modeled using sliding mode design approach. There have been various research-based approaches towards sliding mode controller [29]-[28] towards addressing different phases of research-based problems. Usage of fuzzy logic on sliding mode controller design is carried out by Chen [30] considering problem associated with image stabilization. The work of Rao et al [31] introduced a sliding mode controller 
method by concentrating on the performance enhancement of distributed power flow controller. Similarly, Bouarroudj et al [32] and Othman et al [33], have also adopted sliding mode controller model for non-linear systems and electro-hydrollic actuator system to achieve better control specifications. According to Do [34] identification of non-linear disturbance could improve the controller design. The authors have used similar fuzzy-based sliding mode controller for addressing the chattering effect. Similar fuzzy-based approach was also seen for enhancing power quality.

The work of Elgammal and El-naggar [35] has used this approach for developing active power filter. Similar direction of work has been seen in the work of Fei et al. [36] which witnessed adoption of neural network as well as fuzzy based sliding approach was used for minimizing the chattering effect. Study towards cost optimization using fuzzy-based controller design has been presented by Li et al [37] where the author has typically used interval type-2 fuzzy approach for addressing delay in system. Similar fuzzy approach was also seen in developing sliding window for constructing hybrid controller design with more emphasis on power management issues. The performance of controller design using fuzzy-based sliding window was proven to be enhanced considering case study of vehicle and driving problems. The work of Shen et al [38] has used adaptive mechanism of developing a controller system using same fuzzy-based approach on the top of graph theory.

The work addresses the problems associated with the multi-agent system. The incorporation of adaptiveness is also seen in the work of Wang and Fei [39] where the elimination of chattering has been carried out along with adaptive feature of stability. Wen et al. [40], [41] have developed a mechanism where the dynamic features have been incorporated as well as they have also focused on developing fault tolerant actuator design with enough adaptive features. Another unique form of implementation has been observed in the work of $\mathrm{Yu}$ et al [42] where a bio-inspired algorithm has been designed for better physical control system.

The work carried out by Zhao et al [43] has designed fuzzy controller system considering the case study of closed loop system associated with multi-input and multi-output system. Apart from this there are also other optimization-based approaches towards sliding mode controller design e.g. [44], [45]. Hence, there have been various archives of literatures, where fuzzy-based inference system has been found to be actively used in enhancing the performance of sliding mode converter as well as there are also good number of work being investigated towards navigational approaches too in most recent times. The below section provides the problems evolved in existing system.

\subsection{The Problem}

From the perspective of the advanced navigation system, both INS \& GPS is used in present system. At present majority of the existing navigational system has dependency on GPS however there are various flaws in using GPS e.g. i) abnormal pseudo-range error generation occurred due to tropospheric delay, ionospheres delay any many more. At the same time, usage of INS is also associated with error-prone reading. Following are the evidences to prove this fact about issues of both INS and GPS. The work carried out by Chiang et al [46], Yin et al [47], Filyashkin et al [48], and Han et al [49] are some of the studies where navigational system has been closely studied, however, there are some significant problems associated with all these studies. Some of the potential problems are as follows:

a. Although there are good number of work being carried out in navigational system as well as fuzzy-based sliding mode controller, but they were never being jointly studied. Fuzzy-based sliding approach has been applied on various case studies but not assessed with respect to navigational problems.

b. Existing navigational-based approach doesn't consider complete problems of navigational data that could be possibly arrived from either INS or GPS. It is not clearly mentioned in much of the existing studies that availability of GPS is never confirmed and in such situation user/device will have only impartial or vague navigational data that will lead to error prone directional vectors. Existing approaches towards enhancing precision problems doesn't address this issue.

c. Existing studies towards fuzzy-based sliding mode controllers have used similar architecture of deployment on different case studies. However, it is not considered that there is a fair chance of different applications to possess different requirements of fuzzy structure. Moreover, without improvement of the fuzzy controller, the rule-based approach cannot offer better coverage to scalability factor.

d. The scenarios considered in existing approaches are nearly well-defined with involvement of uncertainty of information from INS. Although, it is a rare case, but it will lead to mal-function of the navigational system that is difficult to be identified. Hence, existing studies are found not to focus on reliability of the inputs considered for navigational data.

Therefore, the prime research problem is that in absence of GPS, INS should offer accurate navigational services with an effective optimized version of fuzzy-based sliding mode controller. This problem is direct representation of dynamic uncertainty that should be better solved by fuzzy logic involved 
in sliding mode controller in vehicle navigational system. The research methodology implemented to overcome these problems is explained with below section.

\section{RESEARCH METHODOLOGY}

The main intention of this work is to design a framework of a novel vehicle navigational system using fuzzy logic and sliding mode controller. However, different from existing approach, the proposed study will choose to initially optimize the error-prone inputs of navigational data followed by applying fuzzy sliding mode controller. The study focuses on developing a novel coupled INS/GPS navigational system along with minimization of chattering problem while using sliding mode controller. The proposed system is performed by analytical research methodology with following scheme as shown in Figure 1.

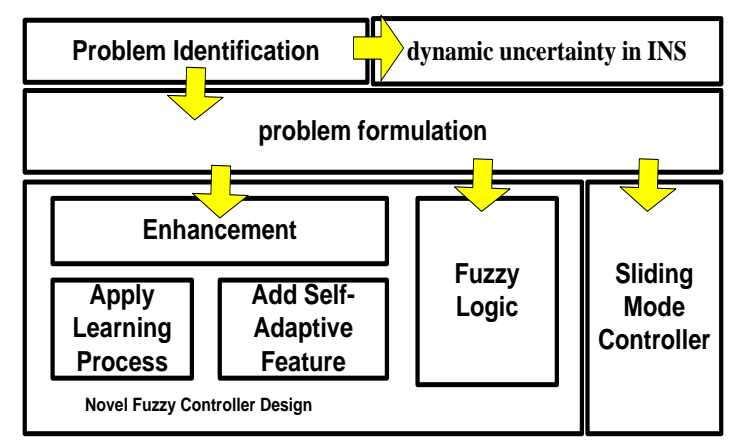

Figure 1. Schematic diagram of 1st methodology

The study considers dynamic uncertainty of time interval of INS when information related to GPS is either incomplete or vague. This dynamic uncertainty will also give rise to maximum non-linear event, which should be suppressed. The initial part of the study will focus on evolving up with a problem formulation related to existing navigational system followed by designing an enhanced fuzzy logic system in order to handle more amounts of uncertainties. This problem is more or less will be given the shape of constraint satisfaction problem The proposed system develops an enhanced fuzzy controller system, where apart from conventional concept of fuzzy logic, it will include designing new capabilities. These capabilities will be formed mathematically considering simple optimization process that will include applying the learning process mentioned by Thangaraj [50]. The study also develop further enhance of this learning process by adding more self-adaptive feature that assists in decision making.

The proposed study also applies stability function in order to assess the stability. The outcome of the study is verified through errors in the signal generated by INS implementing the proposed algorithm as well as speed of the vehicle. The significant contribution of the proposed study are i) performing optimization on navigational data before applying it to fuzzy sliding mode controller, ii) make the design progressive and less iterative by incorporating adaptive features, iii) ensure better response time computationally in order to assess the practical implementation scenario. The next section of the paper discusses about core algorithm implementation in order to achieve the above discussed research methodology.

\section{IMPLEMENTATION OF ALGORITHM}

The designed algorithm is utilized to develop a sophisticated and yet light weight controller system for facilitating better navigational system. However, the proposed algorithm acts very differently from any existing approaches as instead of developing the sliding mode controller, it initially works on optimizing the navigational input. The algorithm for this purpose is highlighted as below:

The proposed system implements a simple optimization technique where the major aim is to obtain better form of structured information about the navigational information. Hence, before developing the proposed form to take the shape of sliding mode fuzzy controller system, the proposed system performs necessary optimization. The first phase of this optimization process is all about initialization process. Hence, a matrix $\mathrm{H}_{\mathrm{a}, \mathrm{b}}$ is constructed randomly by ensuring that there is an undeviating forms of data distribution $\alpha$ (for offering more predictability in navigational data). Hence, for all the $n$ iterations (Line-1), the algorithm obtains the minimal limits of the matrix $H$ i.e. $H_{\text {min,b }}$ where the prefix $a$ and $b$ represents the row and column of the matrix (Line-2). The new variable $\Delta \mathrm{H}$ will represents dot product of an arbitrary generation of 
undeviating form of function and difference of higher and lower bounds of matrix $H$ (Line-2). The proposed system uses probability in this where the numerical value of the undeviating form of function lies in the range of 0 and 1 .

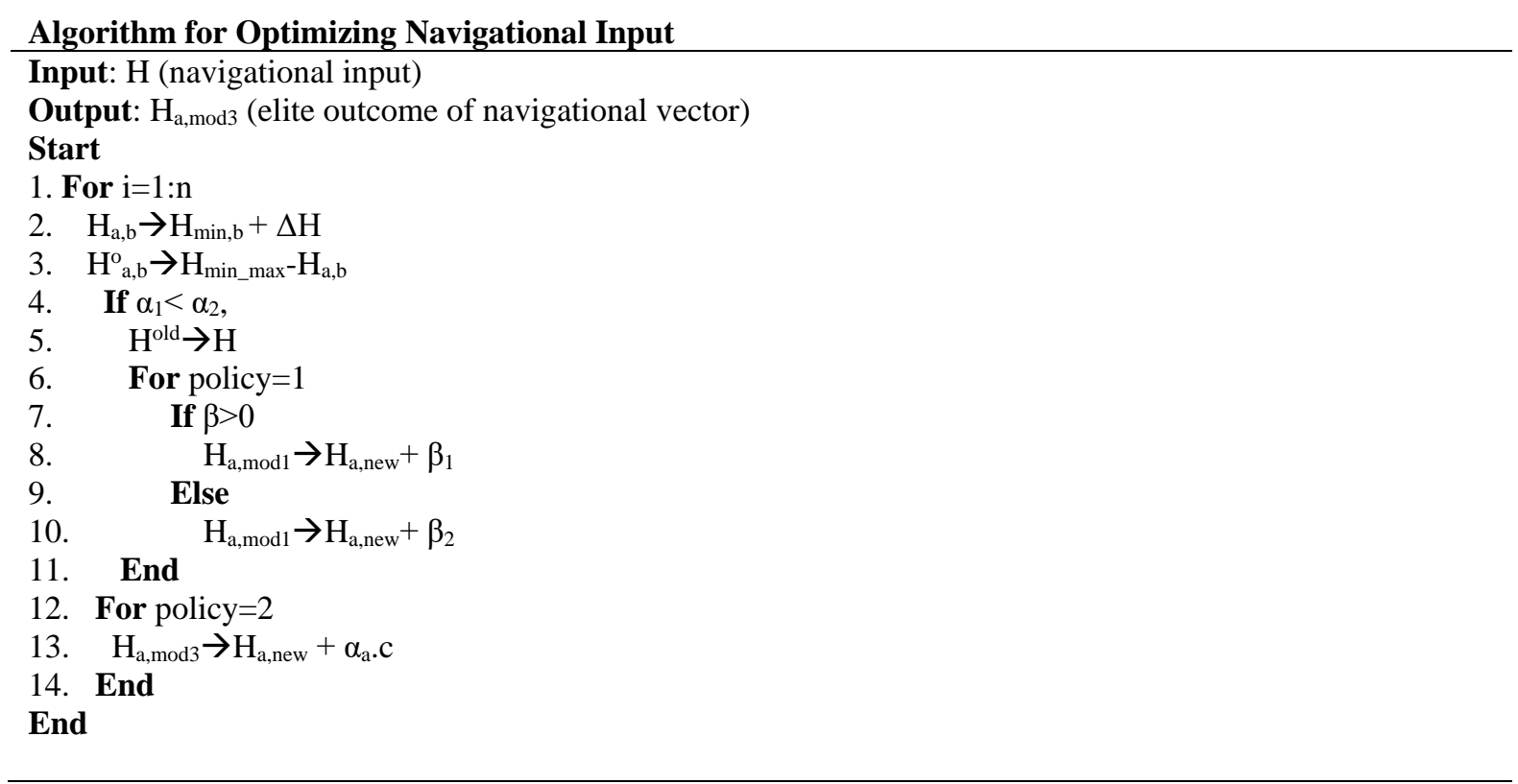

To bring improvement in the convergence speed of the proposed processing, this algorithm further classifies the navigational inputs into clusters. For this purpose, a dynamic system D is formed that is mathematically represented (1),

$$
\mathrm{D}_{\mathrm{a}, \mathrm{b}, \mathrm{c}+1} \rightarrow \theta\left(\pi \mathrm{D}_{\mathrm{c}}\right), c=1,2,3, \ldots c_{\max }
$$

In the above expression, the variable $c$ will represent a recursive function with respect to any trigonometric function $\theta$. The above expression is substituted for undeviating form of function in $\Delta \mathrm{H}$ (Line2). This will finally lead to the normalization of the step (Line-3) to represent the compute value of navigational input $\mathrm{H}_{\text {a,b. }}^{\mathrm{o}}$. The variable $\mathrm{H}_{\text {min_max }}$ will represents bth variable corresponding to the ath array of data in matrix with navigational data. The next part of the algorithm implementation is about formulating a condition for obtaining upgraded data. The algorithm uses a new vector $\mathrm{H}^{\text {old }}$ for performing upgradation of the navigational data in every round. The process of initialization is very similar to $\mathrm{H}$ and the process of upgradation is carried out considering If- THEN which is now termed as policy of upgrading the navigational data for sliding mode fuzzy controller (Line-4).

The empirical relationship between $H_{\text {old }}$ and $H$ is bound by (Line-5). This will also mean that proposed system offers a buffer space where the static information of the navigational data is retained until and unless it is found to be significantly changed. The proposed system performs an arbitrary permutation of the number of navigational input data associated with $H_{\text {old }}$ in order to edit the order to all the unit value of navigational data (Line-5). According to the policy formation, if $\alpha_{1}$ is found to be less than $\alpha_{2}$ than only the upgradation of $H$ will be taken place (Line-5). The next part of the study will further perform second layer of optimization where an adaptive knowledge-acquisition process is initiated. According to this process, the proposed algorithm will be facilitated with better form of decision. For this purpose, the algorithm formulates two different forms of policies.

For the first policy, the algorithm introduces a new variable $\beta$ which is compared with 0 (Line-7), where the comparison results in two different forms of computation of $H$ matrix (Line- 8 and Line-10). The algorithm uses $\beta_{1}$ and $\beta_{2}$ to represents product of $\beta$ with old residual outcome of navigational data (Line- 8 ) and product of $\beta$ with new residual outcome of navigational data (Line-10) respectively. The study considers $\mathrm{H}_{\mathrm{a} \text {,mod } 1}$ to be first elite outcome on the basis of this policy construction. For the second policy formulation, the algorithm implements an empirical expression as shown in Line-13. According to this formulation, the

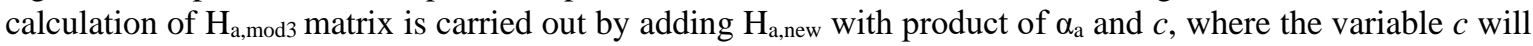
represent a residual outcome of navigational data. In order to obtain residual outcome of navigational data, the recursive function obtains the elite outcome and finds its average. It then subtracts the elite outcome with

Int J Elec \& Comp Eng, Vol. 8, No. 6, December 2018 : 4310 - 4320 
the average score of the navigational data to obtain residual navigational data. The contribution of this algorithm is that implementation of first policy results in building better capabilities for both local as well as global scale of optimization by extracting respective outcomes. However, the second policy assists in improving the global optimization more. Finally, the best policy can be obtained depending upon the outcomes obtained, which is observed by lower score of errors in navigational data. After this part of the optimization is carried out, the next part of the work will be related to applying this optimization principle to the controller design. The mathematical expression for the sliding mode controller system $\rho$ can be now represented as,

$$
\rho(i a) \rightarrow \sum_{i=0}^{n-1} \phi_{1} \cdot \phi_{2}
$$

In the above expression, the variable $i a$ will represents input arguments which is basically an integrated function of error and time. The mathematical expression includes two more variables viz. $\phi_{1}$ and $\phi_{2}$. The first component $\phi_{1}$ represents a constant attributed of positive value and can be represented as,

$$
\phi_{1}=\phi^{\mathrm{c}}
$$

Similarly, the second component $\phi_{2}$ represents an exponential functions whose empirical form is,

$$
\phi_{2}=\mathrm{e}^{\mathrm{n}-\mathrm{q}}
$$

The variable $q$ is equivalent to $(c-1)$ and the proposed study uses a stability function as follows,

$$
S=1 / 2 \rho^{2}
$$

Therefore, the derivative candidate expression for the stability function will be,

$$
S=0.5 \cdot \frac{d \rho^{2}}{d t} \leq-\mu|\rho|
$$

The above expression is basically used for analyzing the amount of stability while designing the controller system, and these further results in

$$
\begin{aligned}
& \rho . \bar{\rho} \leq-\mu|\rho| \\
& \bar{\rho} \cdot \operatorname{sign}(\rho) \leq-\mu
\end{aligned}
$$

The proposed system is said to offer better convergence performance towards the surface of the sliding if the condition $\mu>0$ is found. Even if there are presence of any form of the state-based trajectories than also the proposed system could successfully reach an effective convergence state where $\rho=0$. Hence, now a controller design is expressed empirically as,

$$
\alpha=\delta_{1}-\delta_{2}-\delta_{3}
$$

It can be seen that there are three parameters associated with the controller design. The first parameter $\delta_{1}$ can be formulated as,

$$
\delta_{1}=\frac{1}{k} \cdot K \cdot \alpha
$$

Where, $\mathrm{k} \subseteq \mathrm{K}$ represents gain function. Similarly, the second and third parameter of expression (7) can be represented as,

$$
\delta_{2}=\frac{1}{k} \cdot \chi
$$




$$
\&, \delta_{3}=\frac{1}{k} \cdot \tau(\rho)
$$

The variable $\chi$ and $\tau$ in above expression represents approximating functions and product of gain and $\alpha$ of fuzzy logic respectively considering the input argument of $\rho$. Therefore, it is observed that the proposed system provides a closed-loop system whose stabilization performance is ensured while developing the novel sliding mode fuzzy controller system. This will mean that proposed system offers much reduced dependencies on the completeness of navigational data on some specific instance of $t$ time on uncertain sequences of such occurances. The next section outlines result analysis.

\section{RESULT ANSLYSIS}

As the proposed system introduces a simple and novel sliding mode fuzzy controller system, hence its effectiveness is assessed using speed of the controller and control signal with respect to increasing observation time. The computational effectiveness with viability of the proposed system is analyzed for distribution function and error performance. The outcome is compared with existing system without fuzzy logic implementation (EX-WOF) as well as existing system with fuzzy logic implementation represented as EX-WF [51]. The proposed study uses navigational data [52] to find that proposed system offers better performance.

From the Figure 2 and Figure 3 shows, it is observed that the proposed system provides the higher controller speed and optimal control signal with respect to increasing time. The outcome show that existing mechanism of sliding mode controlling is highly incapable of performing faster convergence to obtain better navigational data and hence its speed is quite lower. Moreover, just by adding fuzzy logic in highly adaptive manner in existing sliding mode controller design only increases the speed to some extent owing to zero optimization on the data, which is carried out in proposed system in the initial stage itself. This is also the prime cause of higher number of control signal from both the forms of existing system while proposed system offers highly predictive control signal irrespective of impartial or vague contents of navigational data.

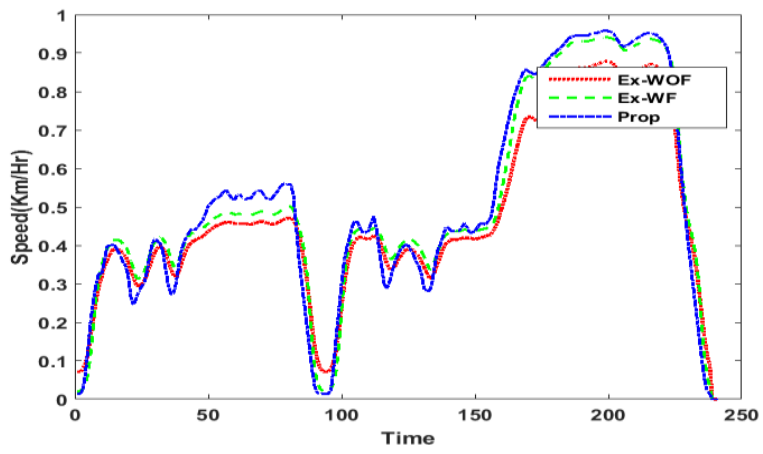

Figure 2. Speed analysis

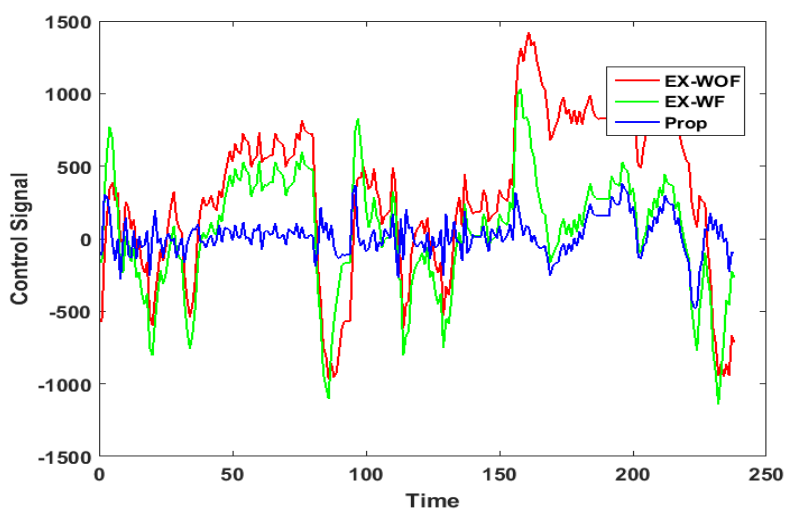

Figure 3. Control Signal analysis 
Figure 4 and Figure 5 highlights the comparative analysis of accuracy with respect to CDF of root mean square error over increasing value of input $\mathrm{H}$ and error performance with different parameters. The outcomes shows that proposed system offers significantly lower scale of error in comparison to existing system. At the same time, it has been observed that proposed system consumes approximately 1.5526 seconds of processing whereas the existing system of both forms consumes approximately 9.3258 seconds. This shows that proposed system offers good accuracy with cost effective computational performance in controller operation.

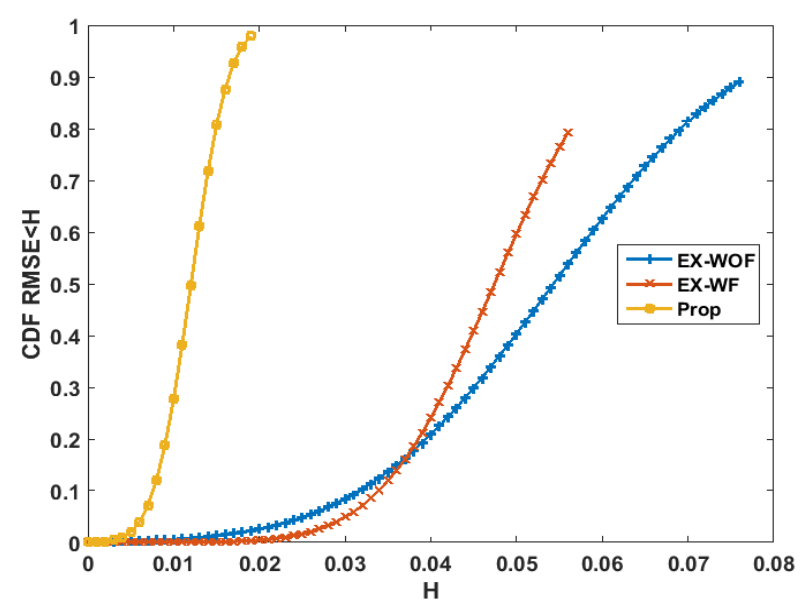

Figure 4. Comparative Analysis of CDF of RMSE

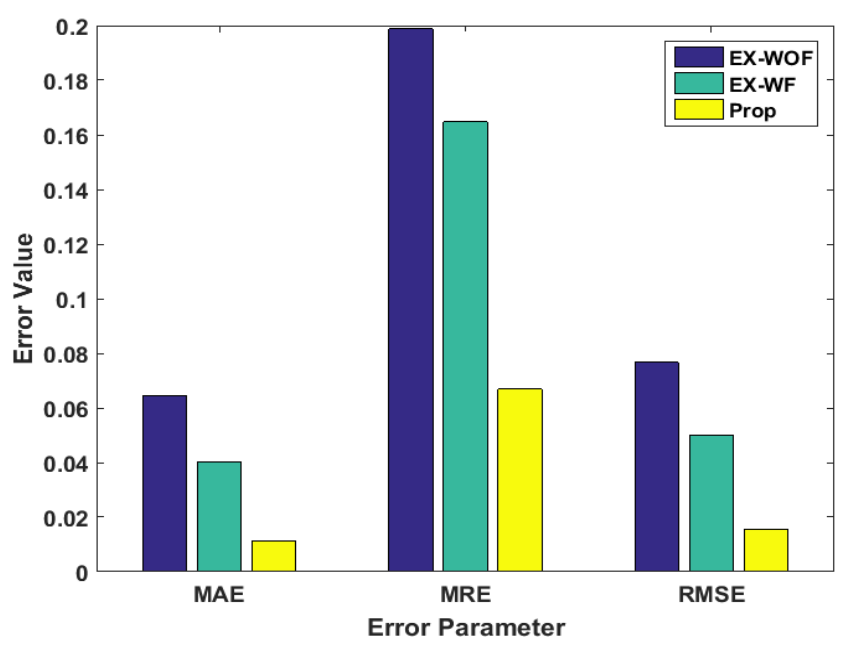

Figure 5. Comparative Analysis of Error Performance

\section{CONCLUSION}

This discussion of paper basically presents a bridge to close the tradeoff of implementing integrated GPS and INS based navigation system with sliding mode controller. The contribution as well as novelty factors of the proposed study are: i) unlike existing approaches of implementing fuzzy sliding mode controller, the proposed system doesn't use fuzzy rules like existing system. The construction of fuzzy rules are created on the basis of accuracy to be obtained in the end stage considering various non-linear parameters, ii) different from existing navigational system, the input data is not processed as it is but it is subjected to optimization using non-linear optimization principle and then it is subjected to fuzzy sliding mode controller, iii) by doing the above two operation, the iterative charecteristics of optimization process is reduced to a large extent to obtained faster convergence performance. The study outcome is found to offer significant improvement in terms of error minimization when compared with existing sliding mode controller design with and without fuzzy approach. 


\section{REFERENCES}

[1] Nilanjan Dey, Amartya Mukherjee, Embedded Systems and Robotics with Open Source Tools, CRC Press, 2016.

[2] Marion Hersh, Michael A Johnson, Assistive Technology for Visually Impaired and Blind People, Springer Science \& Business Media, 2010.

[3] V.E. Balas, L.C. Jain, X. Zhao, Information Technology and Intelligent Transportation Systems, IOS Press, 2017.

[4] Bogusław Dołęga, Robert Głębocki, Damian Kordos, Marcin Żugaj, Advances in Aerospace Guidance, Navigation and Control, 2017.

[5] Wei Quan, Jianli Li, Xiaolin Gong, Jiancheng Fang, INS/CNS/GNSS Integrated Navigation Technology, Springer, 2015.

[6] Stephen W. Hinch, Outdoor Navigation with GPS, Wilderness Press, 2010.

[7] Adam Weintrit, Activities in Navigation: Marine Navigation and Safety of Sea Transportation, CRC Press, 2015

[8] Hassan A. Karimi, Advanced Location-Based Technologies and Services, CRC Press, 2016.

[9] Paul D. Groves, Principles of GNSS, Inertial, and Multisensor Integrated Navigation Systems, Second Edition, Artech House, 2013.

[10] George j. Refuto, Evolution of the US sea-based nuclear missile deterrent: warfighting capabilities, Xlibris Corporation, 2011.

[11] Wilson Wong, James Gordon Fergusson, Military Space Power: A Guide to the Issues, ABC-CLIO, 2010.

[12] Haridwar Singh, Himanshu Shekhar, Solid Rocket Propellants: Science and Technology Challenges, Royal Society of Chemistry, 2016.

[13] P.D. Groves, L. Wang, D. Walter, H. Martin, K. Voutsis and Z. Jiang, "The four key challenges of advanced multisensor navigation and positioning", 2014 IEEE/ION Position, Location and Navigation Symposium - PLANS 2014, Monterey, CA, 2014, pp. 773-792.

[14] Jinkun Liu, Sliding Mode Control Using MATLAB, Academic Press, 2017.

[15] L. Dacheng, L. Zhiguo and Z. Shuai, "Analysis on the influence of the INS precision in an ultra-tightly coupled INS/GPS integration", 2016 IEEE Information Technology, Networking, Electronic and Automation Control Conference, Chongqing, 2016, pp. 1106-1111.

[16] S.Y. Cho, "IM-filter for INS/GPS-integrated navigation system containing low-cost gyros", in IEEE Transactions on Aerospace and Electronic Systems, vol. 50, no. 4, pp. 2619-2629, October 2014.

[17] B.P. Duong and V.H. Nguyen, "Development of a GPS/INS integrated navigation system for model aircraft", 2014 14th International Conference on Control, Automation and Systems (ICCAS 2014), Seoul, 2014, pp. 201-206.

[18] J. Fang and X. Gong, "Predictive Iterated Kalman Filter for INS/GPS Integration and Its Application to SAR Motion Compensation", in IEEE Transactions on Instrumentation and Measurement, vol. 59, no. 4, pp. 909-915, April 2010.

[19] I.U. Lee, H. Li, N.M. Hoang and J.M. Lee, "Navigation system development of the Underwater Vehicles using the GPS / INS sensor fusion", 2014 14th International Conference on Control, Automation and Systems (ICCAS 2014), Seoul, 2014, pp. 610-612

[20] Q. Li and F. Sun, "Strong tracking cubature Kalman filter algorithm for GPS/INS Integrated Navigation System", 2013 IEEE International Conference on Mechatronics and Automation, Takamatsu, 2013, pp. 1113-1117.

[21] Z. Wu, Y. Zhang, J. Sun and Z. Yin, "A filter algorithm for GPS/INS integrated navigation System based on IMMAF", 2016 IEEE International Geoscience and Remote Sensing Symposium (IGARSS), Beijing, 2016, pp. 838-841.

[22] W. Yan, L. Wang, Y. Jin and G. Shi, "High accuracy Navigation System using GPS and INS system integration strategy", 2016 IEEE International Conference on Cyber Technology in Automation, Control, and Intelligent Systems (CYBER), Chengdu, 2016, pp. 365-369.

[23] H. Nakanishi, S. Kanata and T. Sawaragi, "GPS-INS-BARO hybrid navigation system taking into account ground effect for autonomous unmanned helicopter", 2012 IEEE International Symposium on Safety, Security, and Rescue Robotics (SSRR), College Station, TX, 2012, pp. 1-6.

[24] H. Qin, L. Cong and X. Sun, "Accuracy improvement of GPS/MEMS-INS integrated navigation system during GPS signal outage for land vehicle navigation", in Journal of Systems Engineering and Electronics, vol. 23, no. 2, pp. 256-264, April 2012.

[25] Chun-Jung Sun, Chang-Wen Hung, Wei-Cheng Huang and C.E. Lin, "Implementation of GPS/INS navigation system using low-cost MEMS sensors", 2010 5th IEEE Conference on Industrial Electronics and Applications, Taichung, 2010, pp. 1588-1592.

[26] S.Z. Jamal, "Tightly coupled GPS/INS airborne navigation system", in IEEE Aerospace and Electronic Systems Magazine, vol. 27, no. 4, pp. 39-42, April 2012.

[27] M.A.K. Jaradat and M.F. Abdel-Hafez, "Non-Linear Autoregressive Delay-Dependent INS/GPS Navigation System Using Neural Networks", in IEEE Sensors Journal, vol. 17, no. 4, pp. 1105-1115, Feb.15, 152017.

[28] W. Xin, L. Ji, X. Zhang and J. Zhao, "Fault detection and diagnosis in the INS/GPS navigation system", 2014 World Automation Congress (WAC), Waikoloa, HI, 2014, pp. 27-32.

[29] J. Ye, P. Malysz and A. Emadi, "A Fixed-Switching-Frequency Integral Sliding Mode Current Controller for Switched Reluctance Motor Drives", in IEEE Journal of Emerging and Selected Topics in Power Electronics, vol. 3, no. 2, pp. 381-394, June 2015

[30] C.C. Chen, "An optical image stabilization using novel ultrasonic linear motor and fuzzy sliding-mode controller for portable digital camcorders", in IEEE Transactions on Consumer Electronics, vol. 63, no. 4, pp. 343-349, November 2017. 
[31] Rao, D. Narasimha, T. Surnedra, and S. Tara Kalyani, "Improved Performance of DPFC Using Sliding Mode Controller Method", International Journal of Electrical and Computer Engineering, 6.5 (2016): 2073.

[32] Bouarroudj, Noureddine, Djamel Boukhetala, and Fares Boudjema, "Sliding-Mode Controller Based on Fractional Order Calculus for a Class of Nonlinear Systems", International Journal of Electrical and Computer Engineering, 6.5 (2016): 2239.

[33] Othman, Siti Marhainis, et. al., "Optimization of Modified Sliding Mode Controller for an Electro-hydraulic Actuator system with Mismatched Disturbance", International Journal of Electrical and Computer Engineering (IJECE), 8.4 (2018)

[34] T.D. Do, "Disturbance Observer-Based Fuzzy SMC of WECSs Without Wind Speed Measurement", in IEEE Access, vol. 5, pp. 147-155, 2017.

[35] A.A.A. Elgammal and M.F. El-naggar, "MOPSO-based optimal control of shunt active power filter using a variable structure fuzzy logic sliding mode controller for hybrid (FC-PV-Wind-Battery) energy utilisation scheme", in IET Renewable Power Generation, vol. 11, no. 8, pp. 1148-1156, 6282017.

[36] J. Fei, Y. Chu and S. Hou, "A Backstepping Neural Global Sliding Mode Control Using Fuzzy Approximator for Three-Phase Active Power Filter", in IEEE Access, vol. 5, pp. 16021-16032, 2017.

[37] H. Li, J. Wang, L. Wu, H.K. Lam and Y. Gao, "Optimal Guaranteed Cost Sliding-Mode Control of Interval Type-2 Fuzzy Time-Delay Systems", in IEEE Transactions on Fuzzy Systems, vol. 26, no. 1, pp. 246-257, Feb. 2018.

[38] Q. Shen, P. Shi and Y. Shi, "Distributed Adaptive Fuzzy Control for Nonlinear Multiagent Systems Via Sliding Mode Observers", in IEEE Transactions on Cybernetics, vol. 46, no. 12, pp. 3086-3097, Dec. 2016.

[39] T. Wang and J. Fei, "Adaptive Neural Control of Active Power Filter Using Fuzzy Sliding Mode Controller", in IEEE Access, vol. 4, pp. 6816-6822, 2016.

[40] S. Wen, M.Z.Q. Chen, Z. Zeng, X. Yu and T. Huang, "Fuzzy Control for Uncertain Vehicle Active Suspension Systems via Dynamic Sliding-Mode Approach", in IEEE Transactions on Systems, Man, and Cybernetics: Systems, vol. 47, no. 1, pp. 24-32, Jan. 2017.

[41] S. Wen, M.Z.Q. Chen, Z. Zeng, T. Huang and C. Li, "Adaptive Neural-Fuzzy Sliding-Mode Fault-Tolerant Control for Uncertain Nonlinear Systems", in IEEE Transactions on Systems, Man, and Cybernetics: Systems, vol. 47, no. 8, pp. 2268-2278, Aug. 2017.

[42] J. Yu, J. Liu, Z. Wu and H. Fang, "Depth Control of a Bioinspired Robotic Dolphin Based on Sliding-Mode Fuzzy Control Method", in IEEE Transactions on Industrial Electronics, vol. 65, no. 3, pp. 2429-2438, March 2018.

[43] X. Zhao, H. Yang, W. Xia and X. Wang, "Adaptive Fuzzy Hierarchical Sliding-Mode Control for a Class of MIMO Nonlinear Time-Delay Systems With Input Saturation", in IEEE Transactions on Fuzzy Systems, vol. 25, no. 5, pp. 1062-1077, Oct. 2017.

[44] A. Mohanty, S. Patra and P.K. Ray, "Robust fuzzy-sliding mode based UPFC controller for transient stability analysis in autonomous wind-diesel-PV hybrid system", in IET Generation, Transmission \& Distribution, vol. 10, no. 5, pp. 1248-1257, 472016.

[45] S. Rajendiran, P. Lakshmi and B. Rajkumar, "Fractional order fuzzy sliding mode controller for the quarter car with driver model and dual actuators", in IET Electrical Systems in Transportation, vol. 7, no. 2, pp. 145-153, 62017.

[46] Kai-Wei Chiang, Thanh Trung Duong and Jhen-Kai Liao, "The Performance Analysis of a Real-Time Integrated INS/GPS Vehicle Navigation System with Abnormal GPS Measurement Elimination”, Sensor, 2013

[47] Xunyuan Yin, Yingbo Sun, and Changhong Wang, "Positioning Errors Predicting Method of Strapdown Inertial Navigation Systems Based on PSO-SVM", Hindawi, 2013

[48] N.K. Filyashkin and V.S. Yatskivsky, "Prediction of inertial navigation system error dynamics in INS/GPS system", 2013 IEEE 2nd International Conference Actual Problems of Unmanned Air Vehicles Developments Proceedings (APUAVD), Kiev, 2013, pp. 206-209.

[49] Y. Han, M.G. Safonov and J. Chen, "An in-motion alignment method for inertial navigation system based on unfalsified adaptive control theory", Proceedings of 2014 IEEE Chinese Guidance, Navigation and Control Conference, Yantai, 2014, pp. 1900-1905.

[50] R. Thangaraj, M. Pant, T.R. Chelliah and A. Abraham, "Opposition based Chaotic Differential Evolution algorithm for solving global optimization problems", 2012 Fourth World Congress on Nature and Biologically Inspired Computing (NaBIC), Mexico City, 2012, pp. 1-7.

[51] Khooban, M.H., Niknam, T., 'A new and robust control strategy for a class of nonlinear power systems: adaptive general type-II fuzzy’, Proc. Inst. Mech. Eng. I, J. Syst. Control Eng., 2015, 229, (6), pp. 517-528

[52] “U.S. Environmental Protection Agency", https://www.epa.gov/, Retribved on 02 April, 2018

\section{BIOGRAPHIES OF AUTHORS}

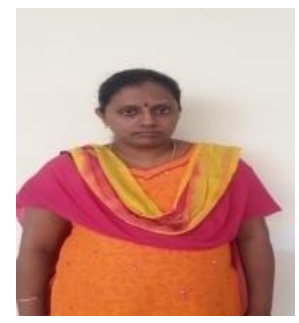

T. Someswari working as assistant professor, EEE dept at The Oxford College of Engineering has published 3 international journal papers. I am pursuing Ph.D in VTU under the guidance of Dr. Anilkumar and Dr. Nagaraj. R. I have completed my B.E degree in 2007 from JNTU, Hydearbad in Electrical and Electronics and M.E from jntu, Anantapur in 2010. 

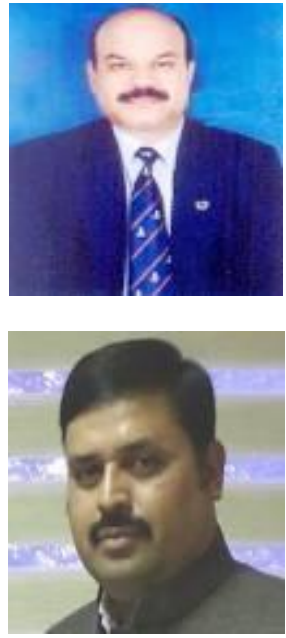

Dr. Anilkumar Tiwari has total of 26 years of experience in Industry, research and teaching (UG \& PG) students I have been worked as Professor \& HOD, Department of ECE in The Oxford College of Engineering, Bangalore Jun 2011 ,Served as Senior PG faculty in Air Force Technical College, Bangalore Jun 2009 -May 2011.Served in Indian Air Force as Senior Engineer super specializing in the areas of Microwave and Radar Engg and Satellite \& Digital Communication, Advanced Missile Guidance and Control Research assignment at Defence Institute of advanced Technology and Defence services staff college. Presently working as Director, Amity school of Engineering.

Dr. Nagaraj R has completed his Ph.D degree from vtu and has published various international and national journals. He has taken his Masters degree from Gulbarga university and B.E from Bangalore university. He has worked as a Director, Dhirubai institute of sciences. Presently, $\mathrm{He}$ is working as a Director, The oxford group of institutions. 